J. Clin. Chem. Clin. Biochem.

Vol. 16, 1978, pp. 293-297

\title{
Elektrolyt- und Kollagengehalt im Rattenherzen bei chronischem Magnesium-Mangel und Streß
}

\author{
Von T. Günther
}

Institut für Molekularbiologie und Biochemie, Freie Universität Berlin,

\section{H. Ising}

Institut für Wasser-, Boden- und Lufthygiene, Bundesgesundheitsamt Berlin und

\section{H. J. Merker}

Anatomisches Institut, Freie Universität Berlin

(Eingegangen am 21. September/12. Dezember 1977)

\section{Herrn Professor Dr. Dr. Ernst Schütte zum 70. Geburtstag gewidmet}

Zusammenfassung: Bei chronischem Mg-Mangel sinkt im Herzmuskel der Gehalt an $\mathrm{Mg}^{\mathbf{1}}$ ) und $\mathrm{K}^{+}$. Der Gehalt an Ca, $\mathrm{Na}^{+}$und Hydroxyprolin nimmt zu. Im Harn werden Adrenalin und besonders Noradrenalin vermehrt ausgeschieden. Gleichzeitiger Lärmstreß verstärkt diese Veränderungen. Die Effekte nehmen mit dem Grade des Mg-Mangels zu. Die Änderungen des $\mathrm{Na}^{+}-, \mathrm{K}^{+}$, $\mathrm{Ca}-, \mathrm{Mg}$ - und Hydroxyprolin-Gehaltes korrelieren mit der Ausscheidung von Noradrenalin. Die Vermehrung des Kollagengehal tes ließ sich elektronenmikroskopisch verifizieren und auf eine Stimulierung von Fibrocyten zurïckführen.

\section{Electrolyte and collagen content of rat heart in chronic Mg-deficiency and stress}

Summary: In chronic Mg-deficiency, there is a decrease in the contents of $\mathrm{Mg}$ and $\mathrm{K}^{+}$in the heart muscle. The contents of $\mathrm{Ca}, \mathrm{Na}^{+}$and hydroxyproline increase. There is also an increased urinary excretion of adrenaline and especially noradrenaline. With simultaneous noise stress, these changes are even greater. The magnitude of the changes increases with the degree of $\mathrm{Mg}$-deficiency. The changes in the contents of $\mathrm{Na}^{+}, \mathrm{K}^{+}, \mathrm{Ca}, \mathrm{Mg}$ and hydroxyproline correlate with the excretion of noradrenaline. The increase in the collagen content can be verified by electron microscopy, and it is due to a stimulation of the fibrocytes.

\section{Einleitung}

An Versuchstieren, bei denen durch Mg-arme Ernährung ein Mg-Mangel erzeugt wurde, treten mannigfaltige Veränderungen auf $(1,2)$. Von den Veränderungen, die sich an den Zellen abspielen, ist primär und im besonderen Maße der Elektrolytstoffwechsel betroffen $(3,4)$. Infolge einer Zunahme der Zellmembranpermeabilität nehmen im Mg-Mangel der zelluläre $\mathrm{K}^{+}$- und Mg-Gehalt $\mathrm{ab}$, während der zellulärre $\mathrm{Na}^{+}$- und Ca-Gehalt zunehmen $(3,4)$. Gleichzeitig erhöht sich im Mg-Mangel der Gehalt an cAMP in verschiedenen Zellarten $(5,6,7)$, besonders im Herzen (5). cAMP bewirkt neben vielen anderen Effekten eine Ëhöhung der Permeabịiliặt (8), ähnlich wie Mg-Mangel.

Andererseits wird die intrazelluläre Konzentration an CAMP durch Catecholamine erhöht, die im Streß vermehrt freigesetżt und ausgeschieden werden. Wenn cAMP an den Wirkungen eines Mg-Mangels beteiligt ist, sollten diese durch Catecholamine bżw. Streß verstärkt werden. Wir haben deshalb die Beziehungen zwischen Catecholaminen sowie Elektrolyt- und Kollagengehalt im Rattenherzen bei verschieden starkem Mg-Mangel und verschieden starkem Lärmstreß im Langzeitversuch untersucht.

\section{Methodik}

Männliche Wistarratten mit einem Gewicht von $150 \mathrm{~g}$ erhielten 16 Wochen lang Mg-armes Futter (Altromin C 1035, Mg-Gehalt: $1,6 \mathrm{mmol} / \mathrm{kg}$ ) und destilliertes Wasser ad libitum. Dem Fútter und Wasser wurde in verschiedenem Maße gemäß Tabelle $1 \mathrm{MgCl}_{2}$ zugegeben.

Tab. 1. Mg-Gehalt der Nahrung

\begin{tabular}{lll}
\hline Gruppe & $\begin{array}{l}\text { Mg-Gehalt } \\
\text { Futter } \\
\text { mmol/kg }\end{array}$ & $\begin{array}{l}\text { Trinkwasser } \\
\text { mmol/1 }\end{array}$ \\
\hline Kontrolle & 83 & 0,4 \\
Mg-Mangei I & 3,8 & 2 \\
Mg-Mangel II & 1,6 & 2 \\
Mg-Mangel III & 1,6 & 0 \\
\hline
\end{tabular}

1) Die Schịeibweise $\mathrm{Mg}^{2+}$ und $\mathrm{Ca}^{2+}$ wurde verwentendet, wenn die freien lonen gemeint sind. $\mathrm{Mg}$ und $\mathrm{Ca}$ stehen für das gesamte (ionisierte und gebundene) Magnesium und Calcium. 
Vier Wochen nach Beginn der Mg-armen Ernährung wurde ein Teil der Ratten in cinem akustischen Spezialraum jeweils während ihrer aktiven Phase von 20.00 bis $8.00 \mathrm{Uhr}$ mit Lärm gestreßt. Als Lärm-Streß wurde auf Magnetband gespeicherter Verkehrslärm verwendet. Der äquivalente Dauerschallpegel (Leq) betrug $69 \mathrm{~dB}(\mathrm{R})(\mathrm{dB}(\mathrm{R})=$ dezi-Bel, bezogen auf die Empfindlichkeit des Rattenohres), der maximale Schallpegel ( Lmax) $86 \mathrm{~dB}(\mathrm{R})$. Beim stärkeren Lärmstreß in einer zweiten Serie wurde zusätzlich ein breitbandiges ständiges Rauschen überlagert (Leq $=73 \mathrm{~dB} .(\mathrm{R}), \mathrm{L} \max =86 \mathrm{~dB}(\mathrm{R})$ ).

In den Gruppen mit leichtem und mittlerem Mg-Mangel (I, II) mit jeweils 4 Tieren überlebten alle Tiere. In der Gruppe mit dem stärksten Mg-Mangel (III) überlebten 5 von 8 Tieren, bei gleichzeitigem Lärmstreß 5 bzw. 4 von je 16 Tieren.

Am Versuchsende wurde den überlebenden Tieren in Äthernarkose Blut und das Herz entnommen. Aus der Vorderwand des linken Ventrikels wurde etwa $1 \mathrm{~mm}^{3}$ für die Elektronenmikroskopie entnommen. Die unteren 3/4 des linken Ventrikels wurden in flüssigem $\mathrm{N}_{2}$ eingefroren und über Silicagel bei 1 Torr gefriergetrocknet und anschließend pulverisiert.

Vom Trockenpulver wurden $\mathrm{Na}^{+}$und $\mathrm{K}^{+}$nach Aufschließen mit konz. $\mathrm{HNO}_{3}$ flammenphotometrisch (Flammenphotometer Eppendorf), Ca und $\mathrm{Mg}$ nach Aufschließen mit konz. $\mathrm{HNO}_{3}-\mathrm{H}_{2} \mathrm{O}_{2}$ im Atomabsorptionsspektrometer (Perkin-Elmer, Modell 300) gemessen.

Die Bestimmung des Hydroxyprolingehaltes erfolgte nach Stegemann (9).

Für die Bestimmung der Adrenalin- und Noradrenalin-Ausscheidung wurde der Harn von jeweils 4 Ratten während der letzten 4 Versuchswochen zwischen 20.00 und $8.00 \mathrm{Uhr}$ gesammelt. In den 12-Stunden-Sammelurinen wurde der Adrenalin- und Noradrenalingehalt fluorimetrisch nach der Trihydroxyindolmethode bestimmt (10). Die Angaben wurden auf den Kreatiningehalt bezogen. Die Bestimmung des Kreatinins erfolgte nach Jaffé gemäß Boehringer-Vorschrift (11).

Für die Elektronenmikroskopie wurden die Proben mit 3\% Paraformaldehyd und 3\% Glutaraldehyd in $0,2 \mathrm{~mol} / 1$ Cacodylatpuffer, pH 7,4, fixiert, in $1 \% \mathrm{OsO}_{4}$ nachfixiert und anschlieBend in Micropal (Ferak, Berlin) eingebettet. Die Schnitte wurden mit LKB- oder Reichert-Mikrotomen hergestellt. Kontrastierung mit Uranylacetat/Bleicitrat. Aufnahmen: Siemens Elmiskop 101.

\section{Ergebnisse und Diskussion}

Wie Tabelle 2 zeigt, führt bei den nicht gestreßten Ratten die Mg-ärmere Ernährung zu einer signifikant geringeren Gewichtszunahme und zu einem signifikant stärkeren Absinken der Mg-Konzentration im Serum. Bei gleichzeitigem Streß ist die Gewichtszunahme, besonders bei der niedrigsten $\mathrm{Mg}$-Zufuhr (III) noch stärker reduziert. Im Streß ändert sich die Mg-Konzentration im Serum nicht mehr parallel mit dem Grade des Mg-Mangels, sondern die Serum-Mg-Konzentration der gestreßten Tiere ist - bei gleichem Mg-Gehalt der Nahrung - höher als bei den ungestreßten Tieren. Als Ursache kommt einmal die geringere Gewichtszunahme im Streß in Frage. Daneben können aber Catecholamine $\mathrm{Mg}$ aus Zellen, besonders aus Muskelzellen (12), freisetzen. Damit übereinstimmend führte Injektion von Adrenalin oder Streß zu einer Zunahme der Mg-Konzentration im Serum (13), und nach Unterbinden der Adrenalinsekretion aus dem Nebennierenmark sank im Mg-Mangel die Serum-MgKonzentration stärker $a b$, als bei vorhandenem Nebennierenmark (12).

Die Tabelle 2 zeigt weiter, daß die für Mg-Mangel typischen Änderungen des zellulären $\mathrm{Na}^{+}, \mathrm{K}^{+}, \mathrm{Ca}$ - und Mg-Gehaltes mit dem Grade des Mg-Mangels zunehmen und durch Streß verstärkt werden. Der Hydroxyprolingehalt im Herzen und die Catecholaminausscheidung im Harn verhielten sich entsprechend.

Die Zunahme der Catecholaminausscheidung betrifft besonders die Ausscheidung von Noradrenalin. Wie Versuche mit adrenalektomierten Ratten ergaben, stammt das ausgeschiedene Adrenalin hauptsächlich aus dem Nebennierenmark, das ausgeschiedene Noradrenalin aus sympathischen Nervenfaserendigungen (14).

Ein Teil der freigesetzten Catecholamine wird wieder zurücktransportiert und in Catecholamin-haltigen Grạnula gespeichert. Der größte Teil wird umgesetzt, d. $h$. oxidiert, methyliert bzw. mit Glucuronsäure oder Sulfat gekoppelt (14). Dennoch geben die Analysen einen Hinweis auf die freigesetzte Menge an Adrenalin und Noradrenalin.

Trägt man dịe Elektrolyt- und Kollagengehalte der einzelnen Versuchsgruppen in Abhängigkeit von der ausgeschiedenen Noradrenalinmenge auf (Abb. 1), so zeigt

Tab. 2. Analysenwerte in Serum, Herz und Harn von normalen Ratten, von Ratten, die mit drei verschiedenen Mg-armen Diäten (I, II, III) ernährt wurden und von Mg-arm ernährten Ratten, die mit zwei verschiedenen Streßarten behandelt wurden. $\overline{\mathrm{x}} \pm s$.

\begin{tabular}{|c|c|c|c|c|c|c|c|c|c|c|}
\hline \multirow[t]{2}{*}{ Tiergruppe } & \multirow[t]{2}{*}{$\mathbf{n}$} & \multirow[t]{2}{*}{ Gewicht } & \multirow{2}{*}{$\begin{array}{l}\text { MgSerum } \\
\text { [mmol/1] }\end{array}$} & $\mathrm{Na}^{+}$ & $\mathrm{K}^{+}$ & $\mathbf{M g}$ & $\mathrm{Ca}$ & $\begin{array}{l}\text { Hydroxÿ- } \\
\text { prolin }\end{array}$ & $\begin{array}{l}\text { Adre- } \\
\text { nalin }\end{array}$ & $\begin{array}{l}\text { Noradre- } \\
\text { nalin }\end{array}$ \\
\hline & & & & \multicolumn{3}{|c|}{ [mmol/kg Trocken-Gewicht] } & & $\begin{array}{l}\text { lg/kg } \\
\mathrm{TG}]^{*}\end{array}$ & \multicolumn{2}{|c|}{$[\mu \mathrm{g} / \mathrm{g} \text { Kreatinin }]^{*}$} \\
\hline $\begin{array}{l}\text { Kontrolle } \\
\text { Mg-Mangel I } \\
\text { Mg-Mangel III }\end{array}$ & $\begin{array}{l}4 \\
4 \\
5\end{array}$ & $\begin{array}{l}402 \pm 8 \\
335 \pm 23 \\
206 \pm 3\end{array}$ & $\begin{array}{l}0,95 \pm 0,03 \\
0,60 \pm 0,02 \\
0,34 \pm 0,01\end{array}$ & $\begin{array}{l}132,0 \pm 1,5 \\
130,6 \pm 1,0 \\
130,5 \pm 3,2\end{array}$ & $\begin{array}{l}269,5 \pm 1,2 \\
268,0 \pm 3,3 \\
259,3 \pm 2,9\end{array}$ & $\begin{array}{l}37,5 \pm 0,8 \\
38,0 \pm 1,7 \\
31,2 \pm 1,4\end{array}$ & $\begin{array}{l}3,0 \pm 0,2 \\
3,5 \pm 0,5 \\
6,2 \pm 0,7\end{array}$ & $\begin{array}{l}3,0 \pm 0,1 \\
3,0 \pm 0,1 \\
3,9 \pm 0,1\end{array}$ & $\begin{array}{l}12 \pm 2 \\
18 \pm 2 \\
20 \pm 5\end{array}$ & $\begin{array}{l}18 \pm 4 \\
23 \pm 4 \\
98 \pm 17\end{array}$ \\
\hline $\begin{array}{l}\text { Mg-Mangel I + Streß } \\
\text { Mg-Mangel II + Streß } \\
\text { Mg-Mangel III + Streß }\end{array}$ & $\begin{array}{l}4 \\
4 \\
5\end{array}$ & $\begin{array}{l}336 \pm 10 \\
287 \pm 18 \\
156 \pm 7\end{array}$ & $\begin{array}{l}0,57 \pm 0,02 \\
0,40 \pm 0,04 \\
0,59 \pm 0,04\end{array}$ & $\begin{array}{l}132,2 \pm 4,3 \\
133,5 \pm 2,0 \\
138,6 \pm 5,5\end{array}$ & $\begin{array}{l}267,3 \pm 3,8 \\
262,7 \pm 2,3 \\
258,7 \pm 2,8\end{array}$ & $\begin{array}{l}37,9 \pm 1,3 \\
34,6 \pm 1,7 \\
29,8 \pm 1,8\end{array}$ & $\begin{array}{l}4,3 \pm 0,2 \\
5,1 \pm 0,7 \\
6,7 \pm 0,6\end{array}$ & $\begin{array}{l}3,0 \pm 0,1 \\
3,3 \pm 0,1 \\
4,6 \pm 0,1\end{array}$ & $\begin{array}{l}16 \pm 2 \\
14 \pm 4 \\
41 \pm 10\end{array}$ & $\begin{array}{r}37 \pm 11 \\
63 \pm 10 \\
129 \pm 19\end{array}$ \\
\hline Mg-Mangel III + Streß 2 & 4 & $145 \pm 6$ & $0,59 \pm 0,05$ & $163,0 \pm 6,0$ & $247,6 \pm 4,5$ & $26,8 \pm 0,8$ & $8,0 \pm 0,9$ & $5,6 \pm 0,9$ & $60 \pm 15$ & $172 \pm 26$ \\
\hline
\end{tabular}

* An Stelle der SI-Einheiten wurden die bisher gebräuchlichen Einheiten gewählt. Aus dem Hydroxyprolingehalt (14\%) ergibt sich somit der Kollagengehalt. 


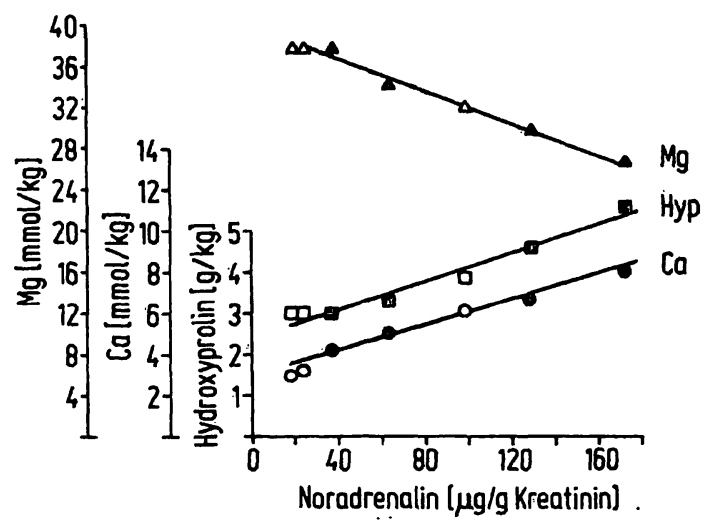

Abb. 1a. Mg-, Ca- und Hydroxyprolin (Hyp)-Gehalt in gefriergetrockneten Rattenherzen (linker Ventrikel) in Abhängigkeit von der Noradrenalinausscheidung.

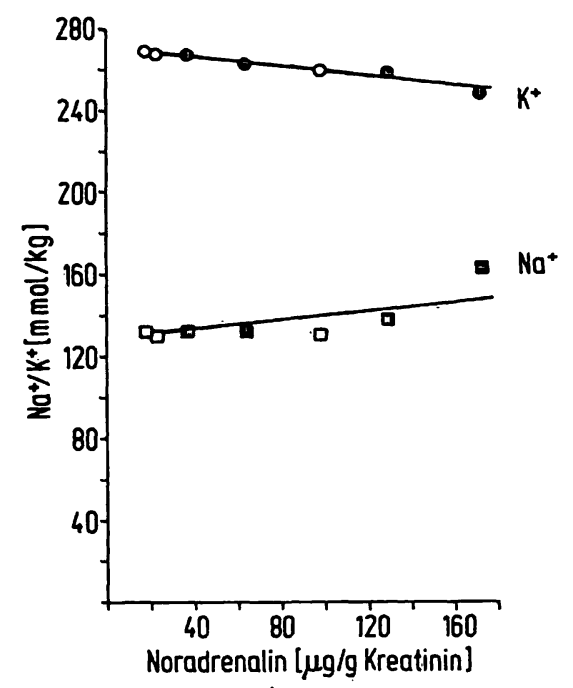

Abb. 1b. $\mathrm{K}^{+}$- und $\mathrm{Na}^{+}-$Gehalt in gefriergetrockneten Rattenherzen wie Abb. 1a. Offene Symbole ohne, ausgefuilte Symbole mit Lärmbehandlung.

sich, daß alle Veränderungen mit der Noradrenalinausscheidung korrelieren. Daraus kann man šchließen, daß die Wirkungen des Mg-Mangels und die Wirkungen von Noradrenalin bžw. Streß funktionell gekoppelt sind und sich deshalb addieren und verstärken. Diese Kopplung kann durch $\mathrm{Mg}^{2+}, \mathrm{Ca}^{2+}$ und/oder cAMP erfolgen.

Catecholamine bewirken einen vermehrten Influx von $\mathrm{Ca}^{2+}$ in die Żellen $(15,16)$, der $\mathrm{z}$. B. durch $\mathrm{Mg}^{2+}$ kompetitiv gehemmt werden kann (16). Im Mg=Mangel nehmen der intrażelluläre $\mathrm{Ca}-$ Gehalt und wahrscheinlich die intrazelluläre $\mathrm{Ca}^{2+}$-Ionenaktivität ebenfalls zu. Catecholamine und Mg-Mangel $(5,6,7)$ bewirken beide einen Anstieg des cAMP. Catecholamine aktivieren bekannntlich nach Bindung an ihren Membranrežeptor die Adenylcyclase.

Mg-Mangel kann auf 2 Arten die Konzentration von cAMP erhöhen:
1. Mg-Mangel führt zu einer vermehrten Freisetzung von Catecholaminen (5) (Tab. 2). Als Mechanismus hierfür kommt die verminderte Serum-Mg-Konzentration im Mg-Mangel in Frage. Dadurch kommt es infolge einer Permeabilitätserhöhung und infolge einer verminderten Konkurrenz zwischen $\mathrm{Mg}^{2+}$ - und $\mathrm{Ca}^{2+}$-Ionen $\mathrm{zu}$ einem vermehrten Influx von $\mathrm{Ca}^{2+}$ in die Catecholaminspeichernden Zellen. Der $\mathrm{Ca}^{2+}$-Influx geht der Catecholaminfreisetzung immer voraus, physiologischerweise infolge einer Permeabilitätssteigerung durch Acetylcholin (17).

2. Mg-Mangel führt auch ohne Beteiligung von Catecholaminen, nachweisbar an isolierten Zellen in der Zellkultur, zum Anstieg von cAMP (6). Hierfür ist wahrscheinlich die Zunahme der intrazellulären $\mathrm{Ca}^{2+}$. Ionenaktivität im Mg-Mangel verantwortlich, wie wir in Versuchen mit dem Ionophor X-537 A an isolierten Zellen zeigen konnten (18). Die (nicht meßbare) intrazelluläre $\mathrm{Ca}^{2+}$-Ionenaktivität könnte sogar ohne Ansteigen des Gesamt-Ca-Gehaltes zunehmen, indem durch die gleichzeitig erhöhte intrazelluläre $\mathrm{Na}^{+}-$Konzentration gespeichertes $\mathrm{Ca}^{2+}$ aus Mitochondrien freigesetzt wird. (19)

Als Mechanismus für die Wirkung des $\mathrm{Ca}^{2+}$ ist eine Aktivierung der Adenylcyclase durch $\mathrm{Ca}^{2+}$ anzunehmen (20). Diese geschieht, indem $\mathrm{Ca}^{2+}$-Ionen an ein $\mathrm{Ca}^{2+}$-bindendes Protein (CDR, Ca-dependent regulator) gebunden werden. Der entstehende Ca-CDR-Komplex aktiviert die Adenylcyclase $(21,22)$. Derselbe Ca-CDRKomplex aktiviert zwar auch die $\mathrm{Ca}^{2+}$-abhängigen Phosphodiesterasen. Diese spalten jedoch vorzugsweise cGMP (23), so daß hiermit auch das oft beobachtete gegensätzliche Verhalten von cAMP und cGMP erklärt werden kann $(23,24)$.

In Tabelle 2 und Abbildung 1 wurde das Verhalten des Hydroxyprolins einbezogen, weil bei chronischem starken Streß eine Vermehrung des Kollagens im Herzen festgestellt wurde (26). Zur Charakterisierung der Hydroxyprolinzunahme wurden Proben aus dem linken Ventrikel elektronenmikroskopisch untersucht. Der Anteil des Kollagens ist im linken Ventrikel niedriger als im rechten (27). Änderungen im Kollagengehalt sind deswegen hier eindeutiger zu sehen. Im Herzen ist das Kollagen besonders im Epicard und Endocard angereichert und folgt dem Lauf der großen Gefaßße.

Abbildung 2 zeigt, daß bei normalen Ratten im interstitiellen Raum zwischen den einzelnen Muskelzellen keine Kollagenfibrillen zu sehen sind. Unter den Bedingungen, die zu einer Zunahme des Hydroxyprolingehaltes führen, sind nunmehr Kollagenfasern im interstitiellen Raum zwischen den Muskelzellen anhand der charakteristischen Querstreifung ihrer Fibrillen nachzuweisen (Abb. 3a, b). Dabei waren keine $\mathrm{Ne}$ krosen auszumachen. 


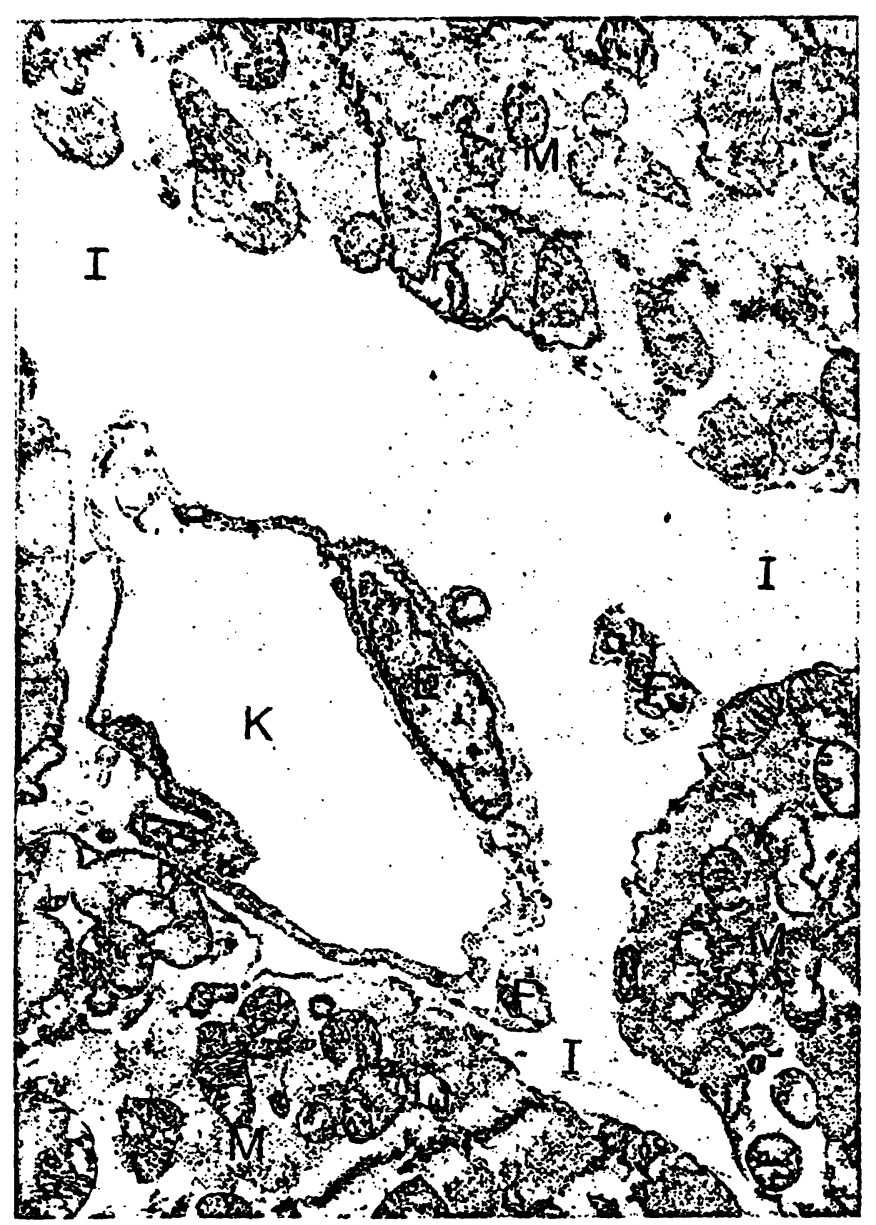

Abb. 2. Elektronenmikroskopische Aufnahme vom linken Myocard einer normalen Ratte. Kapillare (K) mit Endothelzelle (E), F = Fibrocytenfortsatz. Der interstitielle Raum (I) zwischen quergeschnittenen Muskelzellen (M) enthält kein Kollagen. Vergrößerung 1:8000.

Abbildung 4 zeigt einen Fibrocyten-Fortsatz mit einer Vermehrung des rauhen endoplasmatischen Reticulums, an dem die Biosynthese der $\alpha$-Ketten des Prokollagens erfolgt. Für die vermehrte Kollagenbildung sind daher Fibrocyten verantwortlich zu machen. Die Wirkung kann ebenfalls über cAMP erfolgen. Fibrocyten zeigen im Mg-Mangel die gleichen Änderungen im Elektrolytgehalt und im Stoffwechsel (28), wie an Yoshida Ascites Tumorzellen näher untersucht (4). Über einen Anstieg der intrazellulären $\mathrm{Ca}^{2+}$-Konzentration kann es, wie oben beschrieben, in den Fibrocyten zu einem Anstieg des cAMP kommen. Daneben erhöhen aber auch die vermehrt freigesetzten Catecholamine den Gehalt an cAMP in Fibrocyten. In Versuchen mit Fibroblasten-Kulturen wurde nachgewiesen, daß auch Fibroblasten auf Aḋrenalin- und Noradrenalin-Zugabe mit einer Erhöhung des cAMP reagieren (29-31), und nach Zugabe von cAMP kommt es bei Fibroblasten zu einer gesteigerten Synthese von Kollagen $(24,32)$, sowie zu einer vermehrten Freisetzung von Glykosaminoglykanen $(24,33,34)$, den anderen charakteristischen Bestandteilen der bindegewebigen Grundsubstanz.

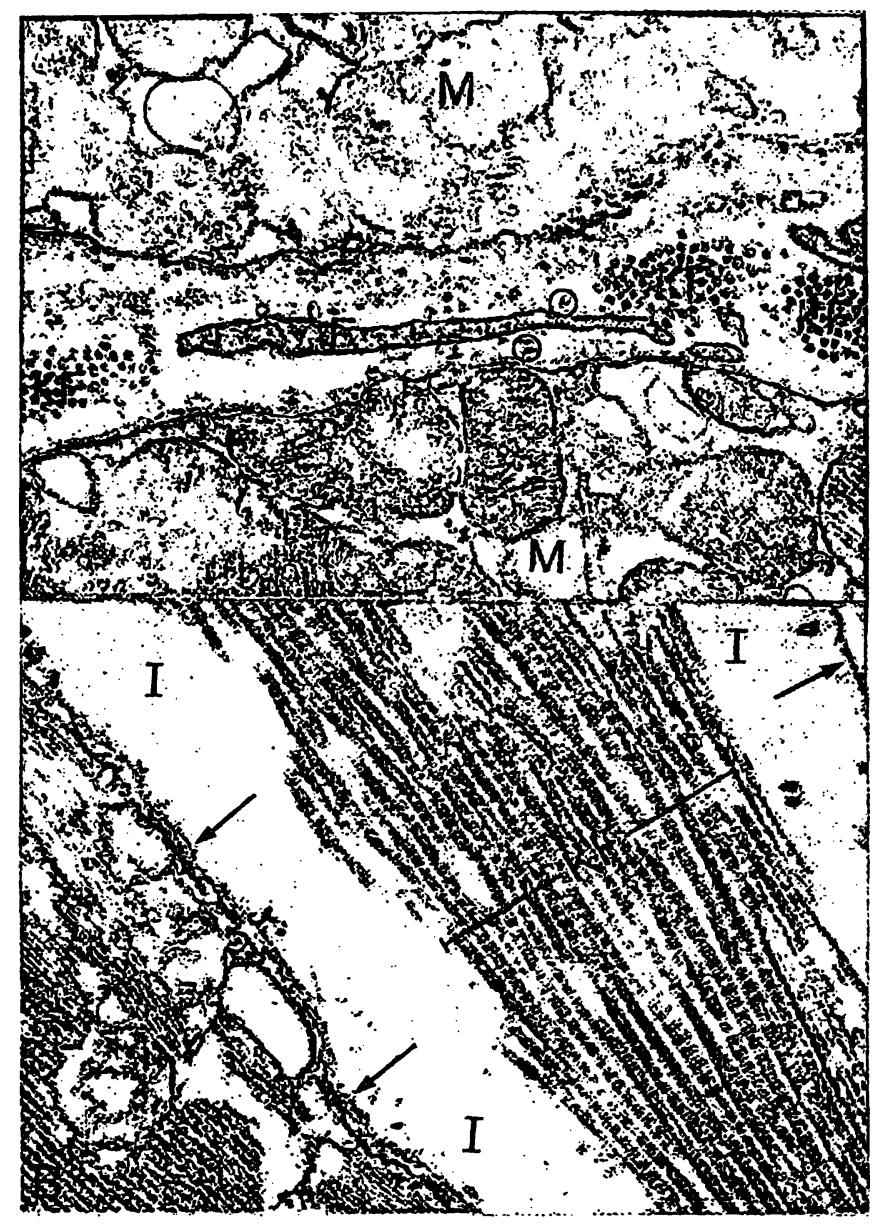

Abb. 3. Elektronenmikroskopische Aufnahme vom linken Myocard einer Mg-arm ernährten Ratte (Mg Mangel II + Streß, Tab. 1, 2), die 12 Wochen mit Lärm (Leq = $69 \mathrm{~dB}(\mathrm{R}), \mathrm{L} \max =86 \mathrm{~dB}(\mathrm{R}))$ gestreßt wurde.

a) Interstitieller Raum zwischen zwei Herzmuskelzellen (M) mit Bündeln von Kollagenfibrillen (KF), einzelnen Kollagenfibrillen (0) und einem angeschnirtenen Fibrocytenfortsatz (F). Vergrößerung 1:27.000.

b) Dickes Bündel aus Kollagenfibrillen (KF) im Interstitium (I) zwischen zwei Herzmuskelzellen (M). Basalmembran ( $\downarrow)$. Vergrößerung 1:29.000.

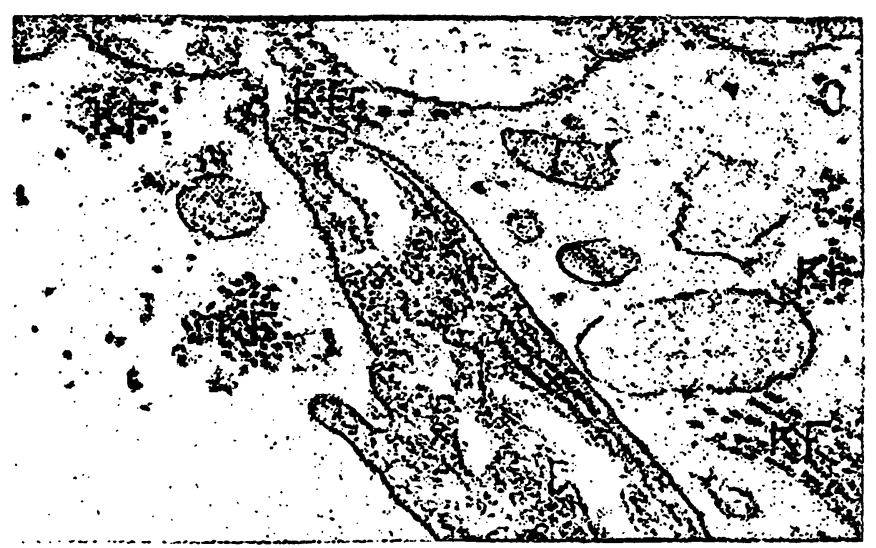

Abb. 4. Elektronenmikroskopische Aufnahme vom linken Myocard einer $\mathrm{Mg}$-arm ernährten Ratte, Gleiche Behandlung wie $A b b$. 3. Fibrocytenfortsatz (F) mit rauhem endoplasmatischem Reticulum (x) in der Nähe einer Muskelzelle $(\mathrm{M})$. Im Interstitium liegen dünne Bündel aus Kollagenfibrillen (KF). Vergrößerung 1:17.000. 


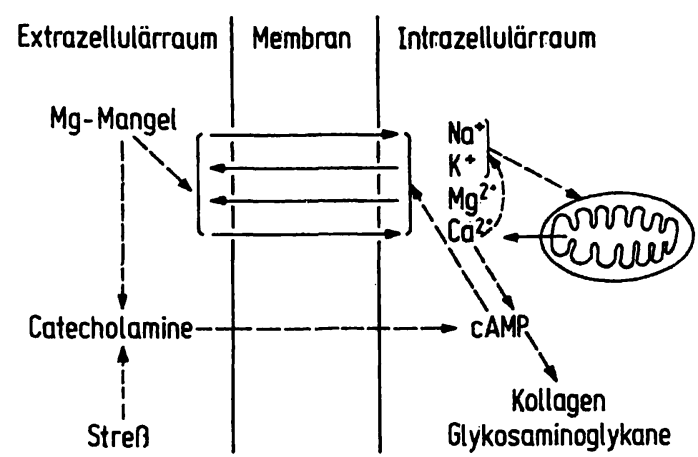

Abb. 5. Biochemische Mechanismen im Mg-Mangel und bei Streß (schematisch). Das Schema bezieht sich auf Fibrocyten, ist aber, wenn man von der Kollagen- und Glyhosaminoglykan-Biosynthese absieht, auf Herzmuskel- und andere Zellen anwendbar.

Im Mg-Mangel nehmen infolge einer Permeabilitätssteigerung der zelluläre $\mathrm{K}^{+}$- und $\mathrm{Mg}$-Gehalt $\mathrm{ab}$, der zelluläre $\mathrm{Na}^{+}$- und $\mathrm{Ca}-\mathrm{Gehalt} \mathrm{zu}$. Die erhöhte intrazelluläre $\left[\mathrm{Na}^{+}\right]$ bewirkt eine Freisetzung von $\mathrm{Ca}^{2+}$ aus Mitochondrien. Die Zunahme der intrazellulären $\left[\mathrm{Ca}^{2+}\right]$ führt zu einer weiteren Permeabilitätssteigerung für $\mathrm{Na}^{+}$und $\mathrm{K}^{+}$sowie über eine Aktivierung der Adenylcy clase zum Ansteigen der [cAMP]. Der erhöhte Konzentration von cAMP bewirkt eine Zunahme der Zellpermeabilität und der Synthese von Kollagen und Glykosaminoglykanen. Mg-Mangel und Streß verursachten eine vermehrte Freisetzung von Catecholaminen, besonders von Noradrenalin. Diese führen über eine Aktivierung der Adenylcyclase zum Ansteigen des cAMP und verstärken auf diese Weise die Wirkungen des Mg-Mangels.

\section{Literatur}

1. Walser, M. (1967), Ergeb. Physiol. Biol. Chem. Exp. Pharmakol. 59, 185-341.

2. Durlach, J. (ed.) (1971), 1. Internat. Sympos. über Magnesium Mangel in der menschlichen Pathologie Vittel, SGEMVVerlag, Vittel.

3. Günther, T. (1969), diese Z. 8, 65-68.

4. Günther, T. \& Averdunk, R. (1970), diese Z. 8, 621-625.

5. Günther, T., Schmalbeck, J. \& Merker, H. J. (1973), diese Z. $11,233-236$.

6. Averdunk, R., Ostapowicz, B. \& Günther, T. (1975), diese Z. 13, 361-366.

7. Rayssiguier, Y., Larvor, P. \& Durlach, J. (1974), C. R. Soc. Biol. 168, 736-738.

8. Friedmann, N. \& Park, C. R. (1968), Proc. Nat. Acad. Sci. $61,504-508$.

9. Stegemann, H. (1958), Hoppe-Seyler's Z. Physiol. Chem. $311,41-45$.

10. Werner, U. (1975), diese Z., 13, 341-349.

11. Testkombination Kreatinin Nr. 124192 , Fa. Boehringer, Mannheim.

12. Lârvor, P. \& Rayssiguier, Y. (1973), in 1. c. (2), S. 473-477.

13. Rayssiguier, Y. \& Larvor, P. (1973), in 1. c. (2), S. 479-484.

14. Documenta Geigy, Georg Thieme Verlagg Stuttgart, 1975, S. $720-724$.

1.5. Reuter, H. \& Scholz, H. (1977), J. Physiol. 264, 49-62.

16. Fleckenstein, A., Janke, J., Frey, M. \& Hein, B. (1977), Arżneimittelforsch. 27, $38^{\prime} \mathrm{L} 389$.

17. Fuller, R. W. (1973), Fed. Proc. 32, 1772-1781.

18. Günther, T. \& Averdunk, R. (1976), diese Z. 14, 365-371.

19. Carafoli, E., Malmström, K., Sigel, E. \& Crompton, M. (1976), Clin. Endocrinol. 5, 49s-59s.

20. Bradham, L. S., Holt, D. A. \& Sims, M. (1970), Biochim. Biophys. Acta, 201, 250-260.

21. Brost.om, G. O., Huang, Y-C., Breckenridge, B. M. \& Wölff, D. J. (1975), Proc. Nat. Acad. Sci., 72, 64-68.
Inwieweit an diesen regulatorischen Wirkungen des cAMP auf die Kollagen- und Glykosaminoglykan- bzw. Proteoglykan-Synthese auch intrazelluläre $\mathrm{Ca}^{2+}$-Ionen beteiligt sind, ist noch zu klären.

Eine Beteiligung von $\mathrm{Mg}^{2+}$-Ionen über eine $\mathrm{Abnahme}$ der intrazellulären $\mathrm{Mg}^{2+}$-Ionenaktivität an den Wirkungen der Catecholamine und des Mg-Mangels ist nicht entschieden, aber wegen der Pufferung der intrazellulären $\mathrm{Mg}^{2+}$-Ionenaktivität $(35,36)$ nicht wahrscheinlich.

In Abbildung 5 sina die Wirkungen des Mg-Mangels und der Catecholamine (Streß) schematisch zusammengefaßt. Das Schema ist, abgesehen von zellspezifischen Eigenschaften, auf verschiedene Zellarten anwendbar. Es können jedoch je nach Ausprägung der Teilfunktionen quantitative Unterschiede bestehen, $z$. B. bedingt durch eine von Zellart zu Zellart unterschiedliche Anzahl an Catecholamin-Rezeptoren, unterschiedliche spezifische Aktivität der Adenylcyclase und unterschiedlicne Membranpermeabilität.

22. Cheung, W. Y., Bradham, L. S., Lynch, T. J., Lin, Y. M. \& Tallant, E. A. (1975), Biochem. Biophys Res. Comm. $66,1055-1062$.

23. Kakiuchi, S., Yamazaki, R., Teshima, Y. \& ULnishi, K. (1973), Proc. Nat. Acad. Sci. 70, 3526-3530.

24. Friedman, D. L. (1976), Physiol. Rev. 56, 652-708.

25. Pastan, I. H., Johnson, G. S. \& Anderson, W. B. (1975), Ann. Rev. Biochem. 44, 491-522.

26. Ising, H., Günther, T., Merker, H. J., Haacke, M. \& Parcell, J. (1976), Zbl. Bakt. Hyg. I. B. 162, 550-557.

27. Caspari, P. G., Gibson, K. \& Harris, P. (1976), in The Biochemistry and Pharmacology of Myocardial Hypertrophy, Hypoxia and Infarction (Harris, P., Bing, R. J. \& Fleckenstein, A., eds.), Urban \& Schwarzenberg, München, Berlin, Wien, S. 99-104.

28. Sanui, H. \& Rubin, H. (1976), J. Cell. Physiol. 92, 23-32.

29. Rao, G. J. S., DelMonte, M. \& Nadler, H. L. (1971), Nature, New Biology, 232, 253-255.

30. Haslan, R. J. \& Goldstein, S. (1974), Biochem. J. 144, 253-263.

31. Kelly, L. A. \& Butcher, R. W. (1974), J. Biol. Chem. 249, 3098-3102.

32. Manner, $r$ \& Kuleba, M. (1974), Connective Tiss. Res. 2, $167-176$.

33. Peters, H. D., Karzel, K., Padberg, D., Schönhöfer, P. S. \& Dinnendahl. V. (1974), Pol. J. Pharmacol. Pharm. 26, 41-49.

34. Shinmei, M., Ghosh, P. \& Taylor, T. K. F. (1976), Biochim. Biophys. Acta 437, 94-105.

35. Günther, T. (1966), Z. Naturforsch. 21b, 1174-1177.

36. Veloso, D., Guynn, R. W., Oskarson, M. \& Veech, R. L. (1973), J. Biol. Chem. 248, 4811-4819.

Prof. Dr. T. Günther Arnimallee 22 1000 Berlin 33 
. , 